\title{
ERGODIC BEHAVIOUR OF NONSTATIONARY REGENERATIVE PROCESSES ${ }^{1}$
}

\author{
BY
}

DAVID MCDONALD

\begin{abstract}
Let $V_{t}$ be a regenerative process whose successive generations are not necessarily identically distributed and let $A$ be a measurable set in the range of $V_{t}$. Let $\mu_{n}$ be the mean length of the $n$th generation and $\alpha_{n}$ be the mean time $V_{t}$ is in $A$ during the $n$th generation. We give conditions ensuring $\lim _{t \rightarrow \infty} \operatorname{Prob}\left\{V_{t} \in A\right\}=\alpha / \mu$ where $\lim _{n \rightarrow \infty}(1 / n) \sum_{j=1}^{n} \mu_{j}=\mu$ and $\lim _{n \rightarrow \infty}(1 / n) \sum_{j=1}^{n} \alpha_{j}=\alpha$.
\end{abstract}

Introduction and main results. A nonstationary regenerative process $V_{t}$ may be viewed as a succession of independent generations or cycles whose cycles are not necessarily identically distributed (a good example is a classical regenerative process (see [8]) whose stochastic mechanism is nonuniformly perturbed). The successive cycles are labelled $1,2,3, \ldots$ and we could start off the process at the $n$th cycle, say, in which case we denote the process by $V_{t}^{(n)}\left(V_{t}^{(1)}\right.$ is denoted by $\left.V_{t}\right)$. However the process is started, it is defined on a measure space $\{\Omega, \mathscr{F}\}$. Starting off at the $n$th cycle merely induces a different probability measure $P^{(n)}$ on $\{\Omega, \mathscr{F}\}\left(P^{(1)}\right.$ is denoted by $\left.P\right)$.

Let $\left(T_{n}\right)_{n=1}^{\infty}$ denote the lengths of the cycles starting at cycle 1 . Hence $\left(T_{n}\right)_{n=1}^{\infty}$ is a sequence of independent random variables defined on $\{\Omega, \mathscr{F}, P\}$. Also we assume $T_{n}>0$ for all $n$. Define $S_{n}=\sum_{m=1}^{n} T_{m}$.

If the $T_{m}$ all take values on a lattice (for simplicity the integers) we call it the lattice case; otherwise we call it the continuous case. As in [2] and [3] we maintain a dichotomy between the two cases. In the former case $R\left[R_{+}\right]$ represents the integers [nonnegative integers]; $B_{+}$is the $\sigma$-field of subsets of $R_{+}$and $m$ is counting measure. In the latter case $R\left[R_{+}\right]$represents $(-\infty, \infty)$ $[[0, \infty)] ; B_{+}$is the $\sigma$-field of Borel sets on $R_{+}$and $m$ is Lebesgue measure.

Let $K_{n}$ be a partition of $\{1,2, \ldots\}$ of the form $K_{n}=\left\{i \mid i_{n}<i<i_{n+1}\right\}$. Given $K_{n}$ define $Y_{n}=\sum_{i \in K_{n}} T_{i}$. For $d>0$, and $\varepsilon=1 / 2 r, r$ an integer, set

Received by the editors August 20, 1976.

AMS (MOS) subject classifications (1970). Primary 60K05, 60K10; Secondary 60F05, 60F99. Key words and phrases. Nonstationary regenerative limits.

${ }^{1}$ This work was done in the author's doctoral thesis at the Université de Montreal under support from the Canada Council. It was completed at Cornell University under a Bourse de Perfectionnement from the Government of Quebec.

(c) 1979 American Mathematical Society 0002-9947/79/0000-0505/\$05.50 


$$
\begin{aligned}
B_{k}(\varepsilon) & =\{x \mid-\varepsilon<x-2 k \varepsilon \leqslant \varepsilon\}, \\
q_{n k}(\varepsilon, d) & =\min \left[\operatorname{Prob}\left\{Y_{n} \in B_{k}(\varepsilon)\right\}, \operatorname{Prob}\left\{Y_{n}-d \in B_{k}(\varepsilon)\right\}\right], \\
q_{n}(\varepsilon, d) & =\sum_{k=-\infty}^{\infty} q_{n k}(\varepsilon, d), \quad Q_{m}=\sum_{n=1}^{m} q_{n}\left(\frac{1}{2}, 1\right) .
\end{aligned}
$$

Definition 1. The sequence $\left\{T_{n}\right\}_{n=1}^{\infty}$ is called strongly $d$-mixing if $\forall \varepsilon$ there exists a sequence $K_{n}$ such that $\sum_{n=0}^{\infty} q_{n}(\varepsilon, d)=\infty$. Furthermore the sequence $\left\{T_{n}\right\}_{n=1}^{\infty}$ is called strongly mixing if the closure of the smallest subgroup containing $\left\{d \mid\left\{T_{n}\right\}_{n=1}^{\infty}\right.$ is strongly $d$-mixing $\}$ is $R$.

Condition $\mathrm{C}(\mathrm{a}) .\left(T_{n}\right)_{n=1}^{\infty}$ is a strongly mixing sequence (see also [2, Definition 3] and also Mineka [4]).

Let $F^{n}$ be the distribution of $T_{n}$.

Condition C(b). There exists a distribution $G$ with finite mean such that $F^{n}(s) \geqslant G(s)$ for all $n$ and all $s \in R_{+}$. Let $\bar{\mu}$ be the mean of $G$.

Condition C(c). If $\mu_{n}=E T_{n}$ is the mean length of the $n$th cycle then inf $\mu_{n} \geqslant \mu>0$. This condition is vacuous in the lattice case.

It is clear that $V_{t}$ is a very special semiregenerative process (see [2] and [3]) with embedded semi-Markov chain $\left(n+1, T_{n}\right)_{n=0}^{\infty}\left(T_{0}=0\right)$. We may therefore apply the ergodic results ([2, Theorem 4] and [3, Theorem 4]) to our special case:

THEOREM 1. If $A$ is a measurable set in the range of $V_{t}$, if Conditions $\mathrm{C}(\mathrm{a})-\mathrm{C}(\mathrm{c})$ hold and if in the continuous case the functions $P^{(n)}\left\{V_{s} \in A\right.$, $\left.X_{1}>s\right\}$ ( $X_{1}$ denotes the length of the starting cycle) are uniformly directly Riemann integrable (see [3, Definition 4]) then

$$
\lim _{\substack{t \rightarrow \infty \\ t \in R_{+}}} \sum_{n=1}^{\infty}\left|P\left\{V_{t} \in A, S_{n-1} \leqslant t<S_{n}\right\}-\frac{\alpha_{n}}{\mu_{n}} P\left\{S_{n-1} \leqslant t<S_{n}\right\}\right|=0
$$

where $\alpha_{n}=\int d P^{(n)} \int_{0}^{X_{1}} \chi_{\left\{V_{s}^{(n)} \in A\right\}}(s) \cdot m(d s)$; that is $\alpha_{n}$ is the mean time $V_{t}$ is in $A$ during the nth cycle.

The goal of this paper is to evaluate the weighting $P\left\{S_{n-1} \leqslant t<S_{n}\right\}$ as $t \rightarrow \infty$. We define the following supplementary conditions:

Let the variance of $F^{n}$ be $\sigma_{n}^{2}$ and set $A_{n}=\sum_{j=1}^{n} \mu_{j}, B_{n}^{2}=\sum_{j=1}^{n} \sigma_{j}^{2}$.

Condition S(a). There exist constants $\underline{\sigma}$ and $\bar{\sigma}$ such that $\sigma_{n}^{2} \leqslant(\bar{\sigma})^{2}$ and $n(\underline{\sigma})^{2} \leqslant B_{n}^{2}$.

CONDITION S(b). $\lim _{b \rightarrow \infty}\left(1 / \sigma_{k}^{2}\right) \int_{|x|<b}\left(x-\mu_{k}\right)^{2} F^{k}(d x)=1$ uniformly in $k$.

Condition S(c). There exists a $\mu>0$ such that $\lim _{n \rightarrow \infty} \sqrt{n}\left(A_{n} / n-\mu\right)=$ 0 .

The chief results of this paper (the proofs are given in the next section) are as follows. 
THEOREM 2. If Conditions $\mathrm{C}(\mathrm{a})-\mathrm{C}(\mathrm{c})$ and $\mathrm{S}(\mathrm{a})-\mathrm{S}(\mathrm{c})$ hold, then

$$
\lim _{\substack{t \rightarrow \infty \\ t \in R_{+}}} \sum_{n=1}^{\infty}\left|P\left\{S_{n-1} \leqslant t<S_{n}\right\}-\frac{\mu_{n}}{\sqrt{2 \pi}} \cdot \frac{1}{B_{n}} \exp \left\{\frac{-(t-n \mu)^{2}}{2 B_{n}^{2}}\right\}\right|=0 \text {. }
$$

Theorem 3. If Conditions $\mathrm{C}(\mathrm{a})-\mathrm{C}(\mathrm{c})$ and $\mathrm{S}(\mathrm{a})-\mathrm{S}(\mathrm{c})$ hold, if (1) holds and if there exists an $\alpha$ such that

$$
\lim _{n \rightarrow \infty} \sqrt{n}\left(\frac{1}{n} \sum_{j=1}^{n} \alpha_{j}-\alpha\right)=0
$$

then

$$
\lim _{\substack{t \rightarrow \infty \\ t \in R_{+}}} P\left\{V_{t} \in A\right\}=\frac{\alpha}{\mu}
$$

This generalizes the classical ergodic result for regenerative processes (see [8]).

Lemmas and proofs. Conditions $\mathrm{C}(\mathrm{a})-\mathrm{C}(\mathrm{c})$ are verified throughout.

LEMMA 1. If Conditions $\mathrm{S}(\mathrm{a})$ are satisfied then for any $\varepsilon>0$ there exists $a \lambda$ such that

$$
\lim _{t \rightarrow \infty} \sum_{\left|\left(t-A_{n}\right) / \sqrt{t}\right|>\lambda} \frac{\mu_{n}}{\sqrt{2 \pi}} \cdot \frac{1}{B_{n}} \exp \left\{\frac{-\left(t-A_{n}\right)^{2}}{2 B_{n}^{2}}\right\}<\varepsilon
$$

ProOF.

$$
\begin{aligned}
& \sum_{\left(t-A_{n}\right) / \sqrt{t}>\lambda} \frac{\mu_{n}}{\sqrt{2 \pi}} \cdot \frac{1}{B_{n}} \exp \left\{\frac{-\left(t-A_{n}\right)^{2}}{2 B_{n}^{2}}\right\} \\
& <\sum_{k=1}^{t / \gamma \underline{\mu}} \frac{\bar{\mu}}{\sqrt{2 \pi}} \cdot \frac{1}{\sqrt{(\underline{\sigma})^{2}((t-\lambda \sqrt{t}) / \bar{\mu}-k)}} \exp \left\{\frac{-(\lambda \sqrt{t}+k \underline{\mu})^{2}}{2(\bar{\sigma})^{2}((t-\lambda \sqrt{t}) / \underline{\mu}-k)}\right\} \\
& \quad+\sum_{k=t / \gamma \mu+1}^{(t-\lambda \sqrt{t}) / \underline{\mu}} \frac{\bar{\mu}}{\sqrt{2 \pi}} \cdot \frac{1}{\underline{\sigma}} \exp \left\{\frac{-(\lambda \sqrt{t}+k \underline{\mu})^{2}}{2(\bar{\sigma})^{2}((t-\lambda \sqrt{t}) / \underline{\mu}-k)}\right\} \\
& \left(\text { where } 0<(1 / \gamma)(\bar{\mu} / \underline{\mu})<1 \text { and } t>\lambda^{2}(1-1 / \gamma)^{-2}\right) \\
& <\frac{(\bar{\mu})^{3 / 2}}{\underline{\sigma}} \cdot \frac{1}{\sqrt{2 \pi}} \int_{0}^{t / \gamma \mu} \frac{1}{\sqrt{t-\lambda \sqrt{t}-x \bar{\mu}} \exp \left\{\frac{-\mu}{2(\bar{\sigma})^{2}} \cdot \frac{(\lambda \sqrt{t}+x \underline{\mu})^{2}}{(t-\lambda \sqrt{t}-x \underline{\mu})}\right\} d x} \\
& +\frac{t}{\underline{\mu}}\left(1-\frac{1}{\gamma}\right) \frac{\bar{\mu}}{\sqrt{2 \pi}} \cdot \frac{1}{\sigma} \exp \left\{\frac{-\mu}{2(\bar{\sigma})^{2}} \cdot \frac{(t / \gamma)^{2}}{t}\right\} .
\end{aligned}
$$


We now choose $0<\alpha<1$ such that

$$
t-\lambda \sqrt{t}-x \bar{\mu}>\alpha(t-\lambda \sqrt{t}-x \underline{\mu})
$$

for $0<x<t / \gamma \mu$. It suffices to find a suitable $\alpha$ for $x=t / \gamma \mu$. For $x=t / \gamma \underline{\mu}$ the above inequality yields $t(1-\alpha-\bar{\mu} / \gamma \mu+\alpha / \gamma)>\lambda \sqrt{t}(1-\alpha)$. Pick $\bar{\alpha}$ so that $\beta=(1-\alpha-\bar{\mu} / \gamma \underline{\mu}+\alpha / \gamma)>0$; that is $0<\alpha<(1-1 / \gamma)^{-1}(1-$ $\bar{\mu} / \gamma \mu)$ and $\beta t>\lambda \sqrt{t}(1-\bar{\alpha})$; that is $t>(\lambda(1-\alpha) / \beta)^{2}$. Therefore for $\alpha, \gamma$ and $\beta$ as above and $t>\max \left\{\lambda^{2}(1-1 / \gamma)^{-2},(\lambda(1-\alpha) / \beta)^{2}\right\}$

$$
\begin{aligned}
& \frac{(\bar{\mu})^{3 / 2}}{\underline{\sigma}} \cdot \frac{1}{\sqrt{2 \pi}} \int_{0}^{t / \gamma \mu} \frac{1}{\sqrt{t-\lambda \sqrt{t}-x \bar{\mu}}} \exp \left\{\frac{-\mu}{2(\bar{\sigma})^{2}} \cdot \frac{(\lambda \sqrt{t}+x \underline{\mu})^{2}}{(t-\lambda \sqrt{t}-x \underline{\mu})}\right\} d x \\
& <\frac{(\bar{\mu})^{3 / 2}}{\underline{\sigma}} \cdot \frac{1}{\sqrt{2 \pi}} \cdot \frac{1}{\sqrt{\alpha}} \int_{0}^{t / \gamma \mu} \frac{1}{\sqrt{t-\lambda \sqrt{t}-x \underline{\mu}}} \\
& \cdot \exp \left\{\frac{-\mu}{2(\bar{\sigma})^{2}} \cdot \frac{(\lambda \sqrt{t}+x \underline{\mu})^{2}}{(t-\lambda \sqrt{t}-x \underline{\mu})}\right\} d x \\
& =\frac{(\bar{\mu})^{3 / 2}}{\underline{\sigma} \underline{\mu}} \cdot \frac{1}{\sqrt{2 \pi}} \cdot \frac{1}{\sqrt{\alpha}} \int_{t(1-1 / \gamma)-\lambda \sqrt{t}}^{t-\lambda \sqrt{t}} \frac{1}{\sqrt{s}} \exp \left\{\frac{-\mu}{2(\bar{\sigma})^{2}} \cdot \frac{(t-s)^{2}}{s}\right\} d s
\end{aligned}
$$

(where $s=t-\lambda \sqrt{t}-x \underline{\mu})$

$$
\begin{array}{rr}
=\frac{(\bar{\mu})^{3 / 2}}{\underline{\sigma} \underline{\mu}} \cdot \frac{1}{\sqrt{2 \pi}} \cdot \frac{1}{\sqrt{\alpha}} \int_{\lambda \sqrt{t}(t-\lambda \sqrt{t})^{-1 / 2}}^{(t / \gamma+\lambda \sqrt{t})(t(1-1 / \gamma)-\lambda \sqrt{t})^{-1 / 2}}\left(1-x\left(x^{2}+4 t\right)^{-1 / 2}\right) \\
\cdot \exp \left\{\frac{-\mu}{(\bar{\sigma})^{2}} \cdot x^{2}\right\} d x
\end{array}
$$

$\left(\right.$ where $x=(t-s) / \sqrt{s}$ or $\left.\sqrt{s}=\left(\sqrt{x^{2}+4 t}-x\right) / 2\right)$

$$
<\frac{(\bar{\mu})^{3 / 2}}{\underline{\sigma} \mu} \cdot \frac{1}{\sqrt{2 \pi}} \cdot \frac{1}{\sqrt{\alpha}} \int_{\lambda}^{\infty} \exp \left\{\frac{-\mu}{(\bar{\sigma})^{2}} x^{2}\right\} d x \text {. }
$$

Therefore,

$$
\begin{aligned}
\sum_{\left(t-A_{n}\right) / \sqrt{t}>\lambda} & \frac{\mu_{n}}{\sqrt{2 \pi}} \cdot \frac{1}{B_{n}} \exp \left\{\frac{-\left(t-A_{n}\right)^{2}}{2 B_{n}^{2}}\right\} \\
< & \frac{(\bar{\mu})^{3 / 2}}{\underline{\sigma} \mu} \cdot \frac{1}{\sqrt{2 \pi}} \cdot \frac{1}{\sqrt{\alpha}} \int_{\lambda}^{\infty} \exp \left\{\frac{-\mu}{(\bar{\sigma})^{2}} x^{2}\right\} d x \\
& +\frac{t}{\underline{\mu}}\left(1-\frac{1}{\gamma}\right) \frac{\bar{\mu}}{\sqrt{2 \pi}} \cdot \frac{1}{\sigma} \exp \left\{\frac{-\mu}{2(\bar{\sigma})^{2} \gamma^{2}} t\right\}<\frac{\varepsilon}{2}
\end{aligned}
$$


for $\lambda$ sufficiently large and $t>\max \left\{\lambda^{2}(1-1 / \gamma)^{-2},\left(\lambda(1-\alpha)^{2} / \beta\right)\right\}$ where $\alpha$, $\lambda$ and $\beta$ are defined as above.

Similarly

$$
\begin{aligned}
& \sum_{\left(t-A_{n}\right) / \sqrt{t}<-\lambda} \frac{\mu_{n}}{\sqrt{2 \pi}} \cdot \frac{1}{B_{n}} \exp \left\{\frac{-\left(t-A_{n}\right)^{2}}{2 B_{n}^{2}}\right\} \\
& <\sum_{k=1}^{\infty} \frac{\bar{\mu}}{\sqrt{2 \pi}} \cdot \frac{1}{\sqrt{(\underline{\sigma})^{2}((t+\lambda \sqrt{t}) / \bar{\mu}+k)}} \exp \left\{\frac{-(\lambda \sqrt{t}+k \underline{\mu})^{2}}{2(\bar{\sigma})^{2}((t+\lambda \sqrt{t}) / \underline{\mu}+k)}\right\} \\
& <\frac{(\bar{\mu})^{3 / 2}}{\underline{\sigma}} \cdot \frac{1}{\sqrt{2 \pi}} \sum_{k=1}^{\infty} \frac{1}{\sqrt{t+\lambda \sqrt{t}+k \underline{\mu}}} \exp \left\{\frac{-\underline{\mu}}{2(\bar{\sigma})^{2}} \cdot \frac{(\lambda \sqrt{t}+k \underline{\mu})^{2}}{(t+\lambda \sqrt{t}+k \underline{\mu})}\right\} \\
& <\frac{(\bar{\mu})^{3 / 2}}{\underline{\sigma}} \cdot \frac{1}{\sqrt{2 \pi}} \int_{0}^{\infty} \frac{1}{\sqrt{t+\lambda \sqrt{t}+x \underline{\mu}}} \exp \left\{\frac{-\mu}{2(\bar{\sigma})^{2}} \cdot \frac{(\lambda \sqrt{t}+x \underline{\mu})^{2}}{(t+\lambda \sqrt{t}+x \underline{\mu})}\right\} \\
& =\frac{(\bar{\mu})^{3 / 2}}{\underline{\sigma \mu}} \cdot \frac{1}{\sqrt{2 \pi}} \int_{t+\lambda \sqrt{t}}^{\infty} \frac{1}{\sqrt{s}} \exp \left\{\frac{-\underline{\mu}}{2(\bar{\sigma})^{2}} \cdot \frac{(s-t)^{2}}{s}\right\} d s
\end{aligned}
$$

(where $s=t+\lambda \sqrt{t}+x \underline{\mu})$

$$
=\frac{(\bar{\mu})^{3 / 2}}{\underline{\sigma} \underline{\mu}} \cdot \frac{1}{\sqrt{2 \pi}} \int_{\lambda \sqrt{t} / \sqrt{t+\lambda \sqrt{t}}}^{\infty}\left(1+\frac{x}{\sqrt{x^{2}+4 t}}\right) \exp \left\{\frac{-\mu}{2(\bar{\sigma})^{2}} \cdot x^{2}\right\} d x
$$

$\left(\right.$ where $x=(t-s) / \sqrt{s}$ or $\left.\sqrt{s}=\left(\sqrt{x^{2}+4 t}+x\right) / 2\right)$

$$
<\frac{(\bar{\mu})^{3 / 2}}{\underline{\sigma} \underline{\mu}} \cdot \frac{1}{\sqrt{2 \pi}} \int_{\lambda}^{\infty} 2 \exp \left\{\frac{-\mu}{2(\bar{\sigma})^{2}} x^{2}\right\} d x<\frac{\varepsilon}{2}
$$

for $\lambda$ sufficiently large.

The result follows.

LEMMA 2. If Conditions $\mathrm{S}(\mathrm{a})$ are verified, then for any $\varepsilon>0$ there exist $\lambda$ and $T(\lambda(\varepsilon))$ such that

$$
\sum_{\left|\left(t-A_{n}\right) / \sqrt{t}\right|>\lambda} P\left\{S_{n-1}<t<S_{n}\right\}<\varepsilon \quad \text { for all } t>T .
$$

ProOF.

$$
\sum_{\left|\left(t-A_{n}\right) / \sqrt{t}\right|>\lambda} P\left(S_{n-1} \leqslant t<S_{n}\right)<P\left(S_{k}>t\right)+P\left(S_{l}<t\right)
$$

where

$$
k=\sup \left\{n: A_{n}<t-\lambda \sqrt{t}\right\}
$$


and

$$
\begin{gathered}
l=\inf \left\{n: A_{n}>t+\lambda \sqrt{t}\right\}, \\
P\left(S_{k}>t\right) \leqslant P\left(S_{k}-A_{k}>\lambda \sqrt{t}\right)<\frac{B_{k}^{2}}{t \lambda^{2}}<\frac{k(\bar{\sigma})^{2}}{t \lambda^{2}} .
\end{gathered}
$$

However $\underline{\mu k}<A_{k}<t-\lambda \sqrt{t}$. Hence

$$
\frac{k}{t}<\frac{1}{\mu}\left(1-\frac{\lambda}{\sqrt{t}}\right)<\frac{1}{\mu} \quad \text { for } t>\lambda^{2} .
$$

Therefore $P\left(S_{k}>t\right)<(1 / \mu)(\bar{\sigma})^{2} / \lambda^{2}$.

$$
P\left(S_{l}<t\right) \leqslant P\left(S_{l}-A_{l}<-\lambda \sqrt{t}\right)<\frac{B_{l}^{2}}{t \lambda^{2}}<\frac{l(\bar{\sigma})^{2}}{t \lambda^{2}} .
$$

However $\underline{\mu}(l-1)<A_{l-1}<t+\lambda \sqrt{t}$. Hence

$$
\frac{l}{t} \leqslant \frac{1}{\underline{\mu}}\left(1+\frac{\lambda}{\sqrt{t}}\right)+\underline{\mu} .
$$

Therefore,

$$
P\left(S_{k}<t\right) \leqslant\left[\left(\frac{1}{\underline{\mu}}+\underline{\mu}\right)+\frac{1}{\underline{\mu}} \cdot \frac{\lambda}{\sqrt{t}}\right] \frac{(\bar{\sigma})^{2}}{\lambda^{2}} .
$$

Hence,

$$
\varlimsup_{t \rightarrow \infty}\left[P\left(S_{k}>t\right)+P\left(S_{l}<t\right)\right]<\frac{(\bar{\sigma})}{\lambda^{2}}\left(\frac{2}{\mu}+\underline{\mu}\right) .
$$

This can be made arbitrarily small by taking $\lambda$ large.

LEMMA 3. If Conditions $\mathrm{S}(\mathrm{a})$ are satisfied then for $L>0, \varepsilon>0, \lambda>0$ there exists $a T>0$ such that for all $t>T$ and for all $x(0<x<L)$ we have

$$
\left|\exp \left\{\frac{-\left(t-x-A_{n}\right)^{2}}{2 B_{n}^{2}}\right\}-\exp \left\{\frac{-\left(t-A_{n+1}\right)^{2}}{2 B_{n+1}^{2}}\right\}\right|<\varepsilon
$$

for all $n$ such that $\left|\left(t-A_{n}\right) / \sqrt{t}\right|<\lambda$.

Proof.

$$
\begin{aligned}
\left|\exp \left\{\frac{-\left(t-x-A_{n}\right)^{2}}{2 B_{n}^{2}}\right\}-\exp \left\{\frac{-\left(t-A_{n+1}\right)^{2}}{2 B_{n+1}^{2}}\right\}\right| \\
<\mid 1-\exp \left\{\frac{\left(t-A_{n+1}\right)^{2}}{2 B_{n+1}^{2}}-\frac{\left(t-x-A_{n}\right)^{2}}{2 B_{n}^{2}}\right\} .
\end{aligned}
$$


Next

$$
\frac{\left(t-A_{n+1}\right)^{2}}{2 B_{n+1}^{2}}-\frac{\left(t-x-A_{n}\right)^{2}}{2 B_{n}^{2}}=\frac{B_{n}^{2}\left(t-A_{n+1}\right)^{2}-B_{n+1}^{2}\left(t-x-A_{n}\right)^{2}}{2 B_{n+1}^{2} B_{n}^{2}} .
$$

Also

$$
\begin{aligned}
B_{n}^{2}\left(t-A_{n}-\mu_{n+1}\right)^{2}- & \left(B_{n}+\sigma_{n+1}^{2}\right)\left(t-x-A_{n}\right)^{2} \\
= & B_{n}^{2}\left(t-A_{n}\right)^{2}-2 B_{n}^{2} \mu_{n+1}\left(t-A_{n}\right)+\left(\mu_{n+1}\right)^{2} B_{n}^{2} \\
& -B_{n}^{2}\left(t-A_{n}\right)^{2}-B_{n}^{2} x^{2}+2 B_{n}^{2} x\left(t-A_{n}\right) \\
& -\sigma_{n+1}^{2}\left(t-A_{n}\right)^{2}+2 x \sigma_{n+1}^{2}\left(t-A_{n}\right)-\sigma_{n+1}^{2} x^{2} .
\end{aligned}
$$

Therefore

$$
\begin{aligned}
\frac{\left(t-A_{n+1}\right)^{2}}{2 B_{n+1}^{2}}- & \frac{\left(t-x-A_{n}\right)^{2}}{2 B_{n}^{2}} \\
= & \frac{-2 \mu_{n+1}\left(t-A_{n}\right)+2 x\left(t-A_{n}\right)}{2 B_{n+1}^{2}}+\frac{\left(\mu_{n+1}\right)^{2}-x^{2}}{2 B_{n+1}^{2}} \\
& +\frac{2 x \sigma_{n+1}^{2}\left(t-A_{n}\right)-\sigma_{n+1}^{2}\left(t-A_{n}\right)^{2}}{2 B_{n}^{2} B_{n+1}^{2}}-\frac{\sigma_{n+1}^{2} x^{2}}{2 B_{n}^{2} B_{n+1}^{2}}
\end{aligned}
$$

as $t \rightarrow \infty, n \rightarrow \infty$. Moreover $B_{n}^{2} \geqslant n(\underline{\sigma})^{2}$ hence

$$
\left\{\left(\mu_{n+1}\right)^{2}-x^{2}\right\} / 2 B_{n+1}^{2} \rightarrow 0, \quad t \rightarrow \infty,
$$

and

$$
-\sigma_{n+1}^{2} x^{2} / 2 B_{n}^{2} B_{n+1}^{2} \rightarrow 0, \quad t \rightarrow \infty,
$$

for $n$ such that $\left|\left(t-A_{n}\right) / \sqrt{t}\right|<\lambda$. Next $A_{n}-\lambda \sqrt{t}<t<A_{n}+\lambda \sqrt{t}$ implies that $t-\lambda \sqrt{t}<n \bar{\mu}$. Hence

$$
\frac{\left|t-A_{n}\right|}{B_{n}^{2}} \leqslant \frac{\dot{\lambda} \sqrt{t}}{B_{n}^{2}} \leqslant \frac{\lambda \sqrt{t}}{n(\underline{\sigma})^{2}} \leqslant \frac{\lambda \sqrt{t} \bar{\mu}}{\underline{\sigma}(t-\lambda \sqrt{t})} .
$$

Therefore $\left|t-A_{n}\right| / B_{n}^{2} \rightarrow 0$ and

$$
\frac{\left(t-A_{n}\right)^{2}}{B_{n}^{2} B_{n+1}^{2}}=\frac{\left(t-A_{n}\right)}{B_{n}^{2}} \cdot \frac{\left(t-A_{n}\right)}{B_{n+1}^{2}} \rightarrow 0
$$

as $t \rightarrow \infty$ by the same reasoning. Hence we have

$$
\frac{\left(t-A_{n+1}\right)^{2}}{2 B_{n+1}^{2}}-\frac{\left(t-x-A_{n}\right)^{2}}{2 B_{n}^{2}} \rightarrow 0
$$

which gives the result. 
Lemma 4. If Conditions $\mathrm{S}(\mathrm{a})$ are verified then $\forall \varepsilon>0, \lambda>0$ there exists a $T>0$ such that for $t>T$

$$
\begin{gathered}
\left|\int_{0}^{t}\left(1-F^{n+1}(t-s)\right) \cdot \frac{1}{\sqrt{2 \pi}} \exp \left\{\frac{-\left(s-A_{n}\right)^{2}}{2 B_{n}^{2}}\right\} m(d s)\right| \\
-\frac{\mu_{n+1}}{\sqrt{2 \pi}} \exp \left\{\frac{-\left(t-A_{n+1}\right)^{2}}{2 B_{n+1}^{2}}\right\} \mid<\varepsilon
\end{gathered}
$$

for $n$ such that $\left|\left(t-A_{n}\right) / \sqrt{t}\right|<\lambda$.

Proof.

$$
\int_{L}^{\infty}\left(1-F^{n}(x)\right) m(d x)<\int_{L}^{\infty}(1-G(x)) m(d x) \rightarrow 0
$$

as $L \rightarrow \infty$. Let $L$ be such that $\int_{L}^{\infty}(1-G(x)) m(d x)<\varepsilon / 4$. Next

$$
\begin{array}{r}
\mid \int_{0}^{t}\left(1-F^{n+1}(t-s)\right)\left[\frac{1}{\sqrt{2 \pi}} \exp \left\{\frac{-\left(s-A_{n}\right)^{2}}{2 B_{n}^{2}}\right\}\right. \\
\left.-\frac{1}{\sqrt{2 \pi}} \exp \left\{\frac{-\left(t-A_{n+1}\right)^{2}}{2 B_{n+1}^{2}}\right\}\right] m(d s) \mid \\
<\int_{0}^{t}(1-G(t-s)) \frac{1}{\sqrt{2 \pi}} \mid \exp \left\{\frac{-\left(s-A_{n}\right)^{2}}{2 B_{n}^{2}}\right\} \\
-\exp \left\{\frac{-\left(t-A_{n+1}\right)^{2}}{2 B_{n+1}^{2}}\right\} \mid m(d s) .
\end{array}
$$

Moreover

$$
\frac{1}{\sqrt{2 \pi}}\left|\exp \left\{\frac{-\left(s-A_{n}\right)^{2}}{2 B_{n}^{2}}\right\}-\exp \left\{\frac{-\left(t-A_{n+1}\right)^{2}}{2 B_{n+1}^{2}}\right\}\right|<1
$$

hence

$$
\begin{gathered}
\int_{0}^{t}(1-G(t-s)) \frac{1}{\sqrt{2 \pi}}\left|\exp \left\{\frac{-\left(s-A_{n}\right)^{2}}{2 B_{n}^{2}}\right\}-\exp \left\{\frac{-\left(t-A_{n+1}\right)^{2}}{2 B_{n+1}^{2}}\right\}\right| m(d s) \\
<\int_{t-L}^{t}(1-G(t-s)) \frac{1}{\sqrt{2 \pi}} \mid \exp \left\{\frac{-\left(s-A_{n}\right)^{2}}{2 B_{n}^{2}}\right\} \\
-\exp \left\{\frac{-\left(t-A_{n+1}\right)^{2}}{2 B_{n+1}^{2}}\right\} \mid m(d s)+\frac{\varepsilon}{4}
\end{gathered}
$$


By Lemma 3 there exists a $T>L$ such that for $t>T$ and $s$ such that $t-L<s<t$ we have

$$
\left|\exp \left\{\frac{-\left(s-A_{n}\right)^{2}}{2 B_{n}^{2}}\right\}-\exp \left\{\frac{-\left(t-A_{n+1}\right)^{2}}{2 B_{n+1}^{2}}\right\}\right|<\frac{\varepsilon}{4 \bar{\mu}}
$$

for $n$ such that $\left|\left(t-A_{n}\right) / \sqrt{t}\right|<\lambda$. Hence we have

$$
\begin{aligned}
\mid \int_{0}^{t}\left(1-F^{n+1}(t-s)\right) & {\left[\frac{1}{\sqrt{2 \pi}} \exp \left\{\frac{-\left(s-A_{n}\right)^{2}}{2 B_{n}^{2}}\right\}\right.} \\
& \left.\quad-\frac{1}{\sqrt{2 \pi}} \exp \left\{\frac{-\left(t-A_{n+1}\right)^{2}}{2 B_{n+1}^{2}}\right\}\right](d s) \mid \\
<\bar{\mu} \cdot \frac{\varepsilon}{4 \bar{\mu}}+\frac{\varepsilon}{4} & =\frac{\varepsilon}{2} .
\end{aligned}
$$

However

$$
\begin{gathered}
\left|\int_{0}^{t}\left(1-F^{n+1}(t-s)\right) m(d s)-\mu_{n+1}\right| \cdot \frac{1}{\sqrt{2 \pi}} \exp \left\{\frac{-\left(t-A_{n+1}\right)^{2}}{2 B_{n+1}^{2}}\right\} \\
<\left|\mu_{n+1}-\int_{0}^{L}\left(1-F^{n+1}(s)\right) m(d s)\right|<\frac{\varepsilon}{2} .
\end{gathered}
$$

This gives the result.

We now digress to establish certain useful results related to local limit theorems.

Proposition 1. In the continuous case suppose $\left\{T_{n}\right\}_{n=1}^{\infty}$ is strongly mixing (we need not assume that the $T_{n}$ are positive valued) and suppose $T_{1}$ has a bounded density; then for all $s, 0<s<l$, where $s \in R_{+}$and $l \in R_{+}$,

$$
\lim _{n \rightarrow \infty} \sum_{k=-\infty}^{\infty} P\left\{k l<S_{n}<k l+s\right\}=\frac{s}{l} \text {. }
$$

PRoof. First suppose $s=x p, l=x q$ where $p$ and $q$ are integers $\left(x \in R_{+}\right)$. Consider the function $f(z)=\chi_{\cup_{k=-\infty}^{\infty}[k l, k l+x)}(z)$. Next let ${ }^{2} S_{n}=\sum_{k=2}^{n} T_{k}$ so

$$
E f\left(S_{n}\right)=E f\left({ }^{2} S_{n}+T_{1}\right)=E \int_{-\infty}^{\infty} f\left({ }^{2} S_{n}+y\right) F^{1}(d y)=E g\left({ }^{2} S_{n}\right)
$$

where

$$
g(x)=\int_{-\infty}^{\infty} f(x+y) F^{1}(d y)
$$

Since $F^{1}$ has a bounded derivative, $g$ is uniformly continuous and applying Theorem 2 in Orey [7] we have:

$$
\lim _{n \rightarrow \infty}\left[E f\left(S_{n}\right)-E f\left(S_{n}+x\right)\right]=\lim _{n \rightarrow \infty}\left[E g\left({ }^{2} S_{n}\right)-E g\left({ }^{2} S_{n}+x\right)\right]=0 .
$$


Similarly

$$
\lim _{n \rightarrow \infty}\left[E f\left(S_{n}\right)-E f\left(S_{n}+k x\right)\right]=0
$$

so

$$
\lim _{n \rightarrow \infty}\left[q E f\left(S_{n}\right)-E \sum_{k=0}^{q-1} f\left(S_{n}+k x\right)\right]=0 ;
$$

that is $\lim _{n \rightarrow \infty}\left[q E f\left(S_{n}\right)-1\right]=0$. Hence $\lim _{n \rightarrow \infty} E \sum_{k=0}^{p-1} f\left(S_{n}+k x\right)=p / q$ which gives:

$$
\lim _{n \rightarrow \infty} \sum_{k=-\infty}^{\infty} P\left\{k l<S_{n}<k l+s\right\}=\frac{s}{l}
$$

if $s / l$ is rational. For arbitrary $s, l$ we can find $S_{L}<s<S_{U}$ such that $S_{L} / l$ and $S_{U} / l$ are rational. Applying the above

$$
\frac{S_{L}}{l} \leqslant \lim _{n \rightarrow \infty} \sum_{k=-\infty}^{\infty} P\left\{k l \leqslant S_{n}<k l+s\right\}
$$

and

$$
\varlimsup_{n \rightarrow \infty} \sum_{k=-\infty}^{\infty} P\left\{k l \leqslant S_{n}<k l+s\right\} \leqslant \frac{S_{U}}{l} .
$$

The result follows by taking $S_{L}$ and $S_{U}$ arbitrarily close to $s$.

The analogous result in the lattice case is obtained similarly. We also have:

Proposition 2. In the continuous case under the hypotheses of Proposition 1 $\lim _{n \rightarrow \infty} \sup _{s \in[\varepsilon, \infty)}\left|\Pi_{k=1}^{n} f_{k}(t)\right|=0$ where $\varepsilon>0$ and $f_{k}(t)$ is the characteristic function of $T_{k}$.

Proof. Let $\bar{T}_{1}$ be a random variable independent of the $T_{k}$ for $k \geqslant 2$ with characteristic function

$$
\bar{f}_{1}(s)= \begin{cases}1-|s| / \varepsilon, & \text { for }|t| \leqslant \varepsilon, \\ 0 & \text { for }|t|>\varepsilon\end{cases}
$$

(see Mineka [5]). Let $\bar{S}_{n}=\bar{T}_{1}+T_{2}+\cdots+T_{n}$. Now $\left(n+1, T_{n}\right)_{n=0}^{\infty}\left(T_{0}=\right.$ $0)$ is a semi-Markov chain with state space $\{1,2, \ldots\}$ and transitions from $n$ to $n+1$ for all $n$. It is clear that all bounded, harmonic functions on the underlying chain are constants. Moreover $\left(n+1, T_{n}\right)_{n=0}^{\infty}$ is strongly mixing. Therefore by Lemma 1(a) in [3], for any harmonic function $h$ on $(n+$ $\left.1, S_{n}\right)_{n=0}^{\infty}, h(n, x)=C_{h}$, a constant, a.e.- $m$ for each $n$. We may consider $\left(n+1, \bar{S}_{n}\right)_{n=0}^{\infty}$ to be the same chain with a different initial measure. By hypothesis both $S_{n}$ and $\bar{S}_{n}$ are absolutely continuous w.r.t. $m$ so, by Theorem 1 in [3], 


$$
\lim _{n \rightarrow \infty}\left\|P\left\{S_{n} \in d x\right\}-P\left\{\bar{S}_{n} \in d x\right\}\right\|=0
$$

( || $\mid$ is the total variation on $R$ ).

Now $E e^{i s \bar{T}_{1}}=0$ for $|s|>\varepsilon$ and hence $E e^{i s \bar{S}_{n}}=0$ for $|s|>\varepsilon$. Hence

$$
\begin{aligned}
\lim _{n \rightarrow \infty} \sup _{s \in[\varepsilon, \infty)}\left|\prod_{k=1}^{n} f_{k}(s)\right| & =\lim _{n \rightarrow \infty} \sup _{s \in[\varepsilon, \infty)}\left|E e^{i s S_{n}}-E e^{i s \bar{S}_{n}}\right| \\
& <\lim _{n \rightarrow \infty}\left\|P\left\{S_{n} \in d x\right\}-P\left\{\bar{S}_{n} \in d x\right\}\right\|=0
\end{aligned}
$$

since $\left|e^{i s x}\right| \leqslant 1$ for all $s$.

Proposition 3. In the lattice case if Condition $\mathrm{C}(\mathrm{a})$ is satisfied then

$$
\prod_{k=1}^{\infty}\left[\max _{0<n<h} P\left\{T_{k}=n(\bmod h)\right\}\right]=0
$$

for any integer $h \geqslant 2$.

Proof. Suppose there exists an integer $\bar{h}$ such that

$$
\prod_{k=1}^{\infty}\left[\max _{0<m<\hbar} P\left\{T_{k}=m(\bmod \bar{h})\right\}\right]>0 .
$$

Then there exists a sequence of integers $\left\{m_{k}\right\}_{k=1}^{\infty}$ such that

$$
\prod_{k=1}^{\infty}\left[P\left\{T_{k}-m_{k}=0(\bmod \bar{h})\right\}\right] \geqslant \varepsilon>0 .
$$

Let $\bar{T}_{k}=T_{k}-m_{k} ; \bar{S}_{n}=\bar{T}_{1}+\bar{T}_{2}+\cdots+\bar{T}_{n}$. Hence $P\left\{\bar{T}_{n}=0(\bmod \bar{h})\right.$ for all $n\}>\varepsilon>0$. Hence $P\left\{\bar{S}_{n}=0(\bmod \bar{h})\right.$ ult $\}>\varepsilon>0$. However the tail field of $\left\{\bar{S}_{n}\right\}_{n=1}^{\infty}$ is clearly contained in the tail field of $\left\{S_{n}\right\}_{n=1}^{\infty}$ which is trivial by hypothesis $\mathrm{C}(\mathrm{a})$. Hence $P\left\{\bar{S}_{n}=0(\bmod \bar{h})\right.$ ult $\}=1$. However

$$
\begin{aligned}
\sum_{i=-\infty}^{\infty} \mid P\{ & \left.S_{n}=i\right\}-P\left\{S_{n}=i+1\right\} \mid \\
& \geqslant\left|P\left\{S_{n}=\sum_{k=1}^{n} m_{k}(\bmod \bar{h})\right\}-P\left\{S_{n}=\sum_{k=1}^{n} m_{k}+1(\bmod \bar{h})\right\}\right| \\
& =\left|P\left\{\bar{S}_{n}=0(\bmod \bar{h})\right\}-P\left\{\bar{S}_{n}=1(\bmod \bar{h})\right\}\right| \\
& \rightarrow 1 \text { as } n \rightarrow \infty
\end{aligned}
$$

since $\bar{S}_{n}=0(\bmod \bar{h})$ ultimately. But by the argument used in Proposition 2 (or by Theorem 2 in [7])

$$
\lim _{n \rightarrow \infty}\left\|P\left\{S_{n} \in d x\right\}-P\left\{S_{n}+1 \in d x\right\}\right\|=0 .
$$

This gives a contradiction and hence completes the proof.

We now state: 
LEMMA 5. If Conditions $\mathrm{C}(\mathrm{a})$ and $\mathrm{S}(\mathrm{a})-\mathrm{S}(\mathrm{b})$ are verified and $T_{1}$ has a bounded density then

$$
\lim _{n \rightarrow \infty}\left|B_{n} p_{n}(s)-\frac{1}{\sqrt{2 \pi}} \exp \left\{\frac{-\left(s-A_{n}\right)^{2}}{2 B_{n}^{2}}\right\}\right|=0
$$

uniformly in $s \in R$ where $p_{n}(s)=P\left\{S_{n}=s\right\}$ in the lattice case and $p_{n}(s)$ is the density of $S_{n}$ in the continuous case.

Proof. It suffices to check the conditions for Theorem 1 in Mineka [5] in the lattice case and Theorem 1 in Muhin [6] in the continuous case. This is easy using Propositions 1-3.

In the discrete case Condition S(b) may be weakened to the Lindeberg condition: for each $\varepsilon>0$

$$
\lim _{n \rightarrow \infty} \frac{1}{B_{n}^{2}} \sum_{k=1}^{n} \int_{|x|>e B_{n}}\left(x-\mu_{k}\right)^{2} F^{k}(d x)=0
$$

plus a stronger mixing condition: $\inf _{n \rightarrow \infty} \sqrt{Q_{n}} / B_{n}>0$. See [1].

LEMMA 6. If Conditions S(a)-S(b) are verified and if $W_{t}(n)=P\left\{S_{n-1}<t<\right.$ $\left.S_{n}\right\}$ then, for all $\lambda>0$,

$$
\lim _{t \rightarrow \infty} \sum_{\left|\left(t-A_{n}\right) / \sqrt{t}\right|<\lambda}\left|W_{t}(n)-\frac{\mu_{n}}{\sqrt{2 \pi}} \cdot \frac{1}{B_{n}} \exp \left\{\frac{-\left(t-A_{n}\right)^{2}}{2 B_{n}^{2}}\right\}\right|=0 .
$$

Proof. By Remark 1 in [3] if $\tilde{T}_{1}$ is a random variable independent of $\left\{T_{n}\right\}_{n=1}^{\infty}$ with bounded derivative then

$$
\lim _{t \rightarrow \infty} \sum_{n=1}^{\infty}\left|W_{t}(n)-P\left\{\tilde{S}_{n-1}<t<\tilde{S}_{n}\right\}\right|=0
$$

where $\tilde{S}_{n}=\tilde{T}_{1}+T_{2}+\cdots+T_{n}$. Hence we may assume that $T_{1}$ has a bounded derivative. Now take $\varepsilon>0$. Next $B_{n}^{2} / n>(\underline{\sigma})^{2}$ for all $n$ as $n \rightarrow \infty$, so there is a $K_{2}$ such that

$$
\sqrt{t / B_{[t / \bar{\mu}-\lambda / \bar{\mu} \sqrt{t}]}^{2}}<k \quad \forall t>K_{2} .
$$

By Lemma 5 there exists an $N$ such that for all $n>N$

$$
\left|B_{n} p_{n}(s)-\frac{1}{\sqrt{2 \pi}} \exp \left\{\frac{-\left(s-A_{n}\right)^{2}}{2 B_{n}^{2}}\right\}\right|<\frac{\varepsilon}{\lambda k \bar{\mu}}
$$

for all $s$. Hence there is a $K_{3}>K_{2}$ such that the inequality $\left|\left(t-A_{n}\right) / \sqrt{t}\right|<$ $\lambda$ implies $n>N$ for $t>K_{3}$. By Lemma 4 there exists a $T>K_{3}$ such that, for $t>T$ 


$$
\begin{aligned}
\mid \int_{0}^{t}\left(1-F^{n+1}(t-s)\right) \frac{1}{\sqrt{2 \pi}} \exp \left\{\frac{-\left(s-A_{n}\right)^{2}}{2 B_{n}^{2}}\right\} m(d s) \\
\quad-\frac{\mu_{n+1}}{\sqrt{2 \pi}} \exp \left\{\frac{-\left(t-A_{n+1}\right)^{2}}{2 B_{n}^{2}}\right\} \mid<\frac{\varepsilon}{\lambda k}
\end{aligned}
$$

for $n$ such that $\left|\left(t-A_{n}\right) / \sqrt{t}\right|<\lambda$. We remark that $W_{t}(n+1)=\int_{0}^{t}(1-$ $\left.F^{n+1}(t-s)\right) p_{n}(s) m(d s)$, so, for $t>T$,

$$
\begin{aligned}
& \sum_{\left|\left(t-A_{n}\right) / \sqrt{t}\right|<\lambda}\left|W_{t}(n+1)-\frac{1}{\sqrt{2 \pi}} \cdot \frac{\mu_{n+1}}{B_{n}} \exp \left\{\frac{-\left(t-A_{n+1}\right)^{2}}{2 B_{n+1}^{2}}\right\}\right| \\
& =\sum_{\left|\left(t-A_{n}\right) / \sqrt{t}\right|<\lambda} \frac{1}{B_{n}} \mid \int_{0}^{t}\left(1-F^{n+1}(t-s)\right) B_{n} p_{n}(s) m(d s) \\
& -\frac{\mu_{n+1}}{\sqrt{2 \pi}} \exp \left\{\frac{-\left(t-A_{n+1}\right)^{2}}{2 B_{n+1}^{2}}\right\} \mid \\
& <\sum_{\left|\left(t-A_{n}\right) / \sqrt{t}\right|<\lambda} \frac{1}{B_{n}} \int_{0}^{t}\left(1-F^{n+1}(t-s)\right) \\
& \cdot\left|B_{n} p_{n}(s)-\frac{1}{\sqrt{2 \pi}} \exp \left\{\frac{-\left(s-A_{n}\right)^{2}}{2 B_{n}^{2}}\right\}\right| m(d s) \\
& +\sum_{\left|\left(t-A_{n}\right) / \sqrt{t}\right|<\lambda} \frac{1}{B_{n}} \mid \int_{0}^{t}\left(1-F^{n+1}(t-s)\right) \frac{1}{\sqrt{2 \pi}} \\
& \cdot \exp \left\{\frac{-\left(s-A_{n}\right)^{2}}{2 B_{n}^{2}}\right\} m(d s) \\
& -\frac{\mu_{n+1}}{\sqrt{2 \pi}} \exp \left\{\frac{-\left(t-A_{n+1}\right)^{2}}{2 B_{n+1}^{2}}\right\} \mid \\
& <\sum_{\left|\left(t-A_{n}\right) / \sqrt{t}\right|<\lambda} \frac{1}{B_{n}} \bar{\mu} \cdot \frac{\varepsilon}{\lambda k \bar{\mu}} \text { (by (3)) } \\
& +\sum_{\left|\left(t-A_{n}\right) / \sqrt{t}\right|<\lambda} \frac{1}{B_{n}} \frac{\varepsilon}{\lambda k} \quad \text { (by (4)) } \\
& <\frac{2 \varepsilon}{\lambda k} \cdot \frac{1}{B_{[t / \bar{\mu}-\lambda \sqrt{t} / \bar{\mu}]}} \cdot \frac{2 \lambda}{\underline{\mu}} \sqrt{t} \text {, }
\end{aligned}
$$


using $n \bar{\mu}>t-\lambda \sqrt{t}$. But $t>T>K_{2}$ so $\sqrt{t} / B_{[t / \bar{\mu}-\lambda \sqrt{t} / \bar{\mu}]}<k$. Therefore we have

$$
\sum_{\left|\left(t-A_{n}\right) / \sqrt{t}\right|<\lambda}\left|W_{t}(n+1)-\frac{1}{\sqrt{2 \pi}} \cdot \frac{\mu_{n+1}}{B_{n}} \exp \left\{\frac{-\left(t-A_{n+1}\right)^{2}}{2 B_{n+1}^{2}}\right\}\right|<\frac{4 \varepsilon}{\underline{\mu}} .
$$

Finally

$$
\begin{gathered}
\sum_{\left|\left(t-A_{n}\right) / \sqrt{t}\right|<\lambda} \frac{1}{\sqrt{2 \pi}} \mu_{n+1} \\
\exp \left\{\frac{-\left(t-A_{n+1}\right)^{2}}{2 B_{n+1}^{2}}\right\}\left|\frac{1}{B_{n}}-\frac{1}{B_{n+1}}\right| \\
\leqslant 2 \frac{\lambda}{\mu} \sqrt{t} \frac{\sigma_{G}}{B_{n-1} B_{n}}
\end{gathered}
$$

since $\sqrt{B_{n+1}^{2}-B_{n}^{2}} \geqslant B_{n+1}-B_{n}$. This tends to 0 as $t \rightarrow \infty$. We remark that

$$
W_{t}([t / \bar{\mu}+\lambda \sqrt{t} / \bar{\mu}]) \rightarrow 0 \quad \text { as } t \rightarrow \infty
$$

which implies

$$
\sum_{\left|\left(t-A_{n}\right) / \sqrt{t}\right|<\lambda}\left|W_{t}(n)-\frac{1}{\sqrt{2 \pi}} \cdot \frac{\mu_{n}}{B_{n}} \exp \left\{\frac{-\left(t-A_{n}\right)^{2}}{2 B_{n}^{2}}\right\}\right|<4 \varepsilon
$$

which yields the result.

Proposition 1. If Conditions S(a)-S(b) are verified then

$$
\lim _{t \rightarrow \infty} \sum_{n=1}^{\infty}\left|W_{t}(n)-\frac{\mu_{n}}{\sqrt{2 \pi}} \cdot \frac{1}{B_{n}} \exp \left\{\frac{-\left(t-A_{n}\right)^{2}}{2 B_{n}^{2}}\right\}\right|=0 .
$$

Proof. This is immediate from Lemmas 1, 2 and 6.

Remarks. Proposition 1 gives

$$
\lim _{t \rightarrow \infty} \sum_{n=1}^{\infty} \frac{\mu_{n}}{\sqrt{2 \pi}} \cdot \frac{1}{B_{n}} \exp \left\{\frac{-\left(t-A_{n}\right)^{2}}{2 B_{n}^{2}}\right\}=\lim _{t \rightarrow \infty} \sum_{n=1}^{\infty} W_{t}(n)=1 .
$$

LEMMA 7. If Conditions S(a)-S(c) are satisfied,

$$
\begin{aligned}
& \lim _{t \rightarrow \infty} \sum_{\left|\left(t-A_{n}\right) / \sqrt{t}\right|<\lambda} \mid \frac{1}{\sqrt{2 \pi}} \cdot \frac{1}{B_{n}} \exp \left\{\frac{-\left(t-A_{n}\right)^{2}}{2 B_{n}^{2}}\right\} \\
&-\frac{1}{\sqrt{2 \pi}} \cdot \frac{1}{B_{n}} \exp \left\{\frac{-(t-n \mu)^{2}}{2 B_{n}^{2}}\right\} \mid=0 .
\end{aligned}
$$


Proof.

$$
\begin{aligned}
& \sum_{\left|\left(t-A_{n}\right) / \sqrt{t}\right|<\lambda} \mid \frac{1}{\sqrt{2 \pi}} \cdot \frac{1}{B_{n}} \exp \left\{\frac{-\left(t-A_{n}\right)^{2}}{2 B_{n}^{2}}\right\} \\
&-\frac{1}{\sqrt{2 \pi}} \cdot \frac{1}{B_{n}} \exp \left\{\frac{-(t-n \mu)^{2}}{2 B_{n}^{2}}\right\} \mid \\
&<\quad \sum_{n} \frac{1}{\sqrt{2 \pi}} \cdot \frac{1}{B_{n}} \exp \left\{\frac{-\left(t-A_{n}\right)^{2}}{2 B_{n}^{2}}\right\} \\
& \cdot\left|1-\exp \left\{\frac{-2 t A_{n}+A_{n}^{2}+2 t n \mu-n^{2} \mu^{2}}{2 B_{n}^{2}}\right\}\right| .
\end{aligned}
$$

However

$$
\frac{-2 t A_{n}+A_{n}^{2}+2 t n \mu-n^{2} \mu^{2}}{2 B_{n}^{2}}=-\frac{\left[A_{n}-n \mu\right]}{2 B_{n}} \cdot\left[\frac{2\left(t-A_{n}\right)}{B_{n}}+\frac{A_{n}-n \mu}{B_{n}}\right] .
$$

But

$$
\frac{A_{n}-n \mu}{B_{n}}=\frac{A_{n}-n \mu}{\sqrt{n}} \cdot \frac{\sqrt{n}}{B_{n}} \rightarrow 0 \quad \text { as } n \rightarrow \infty .
$$

Also $\left|\left(t-A_{n}\right) / \sqrt{t}\right|<\lambda$ implies $\sqrt{n}>((t-\lambda \sqrt{t}) / \bar{\mu})^{1 / 2}$, hence

$$
\left|\frac{t-A_{n}}{\sqrt{n}}\right|<\frac{\lambda \sqrt{t}}{\sqrt{n}} \leqslant \lambda \sqrt{t}\left(\frac{t-\lambda \sqrt{t}}{\bar{\mu}}\right)^{-1 / 2} \text { if }\left|\frac{t-A_{n}}{\sqrt{t}}\right|<\lambda
$$

$<k$ for all $t$, for some constant $k$.

Therefore $(t-n \mu) / B_{n}=O(1)$. Hence for any $\varepsilon>0$, we may choose a $T>0$ such that for $t>T$

$$
\left|\exp \left\{\frac{-2 t A_{n}+A_{n}^{2}+2 t n \mu-n^{2} \mu^{2}}{2 B_{n}^{2}}\right\}-1\right|<\varepsilon
$$

for all $n$ such that $\left|\left(t-A_{n}\right) / \sqrt{t}\right|<\lambda$. Therefore

$$
\begin{aligned}
\varlimsup_{\left|\left(t-A_{n}\right) / \sqrt{t}\right|<\lambda} & \frac{1}{\sqrt{2 \pi}} \cdot \frac{1}{B_{n}} \exp \left\{\frac{-\left(t-A_{n}\right)^{2}}{2 B_{n}^{2}}\right\} \\
& -\frac{1}{\sqrt{2 \pi}} \cdot \frac{1}{B_{n}} \exp \left\{\frac{-(t-n \mu)^{2}}{2 B_{n}^{2}}\right\} \mid \\
& <\varepsilon \varlimsup_{t \rightarrow \infty} \sum_{\left|\left(t-A_{n}\right) / \sqrt{t}\right|<\lambda} \frac{1}{\sqrt{2 \pi}} \cdot \frac{1}{B_{n}} \exp \left\{\frac{-\left(t-A_{n}\right)^{2}}{2 B_{n}^{2}}\right\} .
\end{aligned}
$$


Now

$$
\varlimsup_{t \rightarrow \infty} \sum_{\left|\left(t-A_{n}\right) / \sqrt{t}\right|<\lambda} \frac{1}{\sqrt{2 \pi}} \cdot \frac{1}{B_{n}} \exp \left\{\frac{-\left(t-A_{n}\right)^{2}}{2 B_{n}^{2}}\right\}<1 .
$$

The result follows.

LEMMA 8. If Conditions S(a)-S(c) are satisfied then for any $\varepsilon>0$ there exists a $\lambda_{2}$ such that

$$
\lim _{t \rightarrow \infty} \sum_{|(t-n \mu) / \sqrt{t}|>\lambda_{2}} \frac{\mu_{n}}{\sqrt{2 \pi}} \cdot \frac{1}{B_{n}} \exp \left\{\frac{-(t-n \mu)^{2}}{2 B_{n}^{2}}\right\}<\varepsilon .
$$

Proof. This follows from Lemma 1.

Note that

$$
\frac{1}{\sqrt{2 \pi} \sigma} \int_{-\infty}^{\infty} e^{-\left(\mu^{2} / 2 \sigma^{2}\right) s^{2}} d s=\frac{1}{\mu}
$$

Hence there is a $\bar{\lambda}>0$ such that

$$
\left|\frac{1}{\sqrt{2 \pi} \sigma} \int_{-\lambda / \sqrt{\mu}}^{\lambda / \sqrt{\mu}} e^{-\left(\mu^{2} / 2 \sigma^{2}\right) s^{2}} d s-\frac{1}{\mu}\right|<\tilde{\varepsilon} .
$$

If we break $[-\bar{\lambda} / \sqrt{\mu}, \bar{\lambda} / \sqrt{\mu}]$ at the following points:

$$
\left\{\ldots, \frac{-n}{\sqrt{\bar{n}-n}}, \ldots, \frac{-1}{\sqrt{\bar{n}-1}}, 0, \frac{1}{\sqrt{\bar{n}+1}}, \ldots, \frac{n}{\sqrt{\bar{n}+n}}, \ldots\right\},
$$

we have

$$
\begin{aligned}
\frac{1}{\sqrt{2 \pi} \sigma} \int_{-\lambda / \sqrt{\mu}}^{\pi / \sqrt{\mu}} e^{-\left(\mu^{2} / 2 \sigma^{2}\right) s^{2} d s=} & \lim _{n \rightarrow \infty} \sum_{|n|<\lambda \sqrt{n / \mu}} \frac{1}{\sqrt{2 \pi \sigma^{2}}} \\
& \cdot \frac{1}{\sqrt{\bar{n}+n}} \exp \left\{-\frac{1}{2} \frac{\mu^{2}}{\sigma^{2}}\left(\frac{n}{\sqrt{\bar{n}+n}}\right)^{2}\right\}
\end{aligned}
$$

since $(n+1) / \sqrt{\bar{n}+n+1}-n / \sqrt{\bar{n}+n} \approx 1 / \sqrt{\bar{n}+n}$. Let $\mu \bar{n}=t$ :

$$
\begin{aligned}
\frac{1}{\sqrt{2 \pi \sigma}} \int_{-\lambda / \sqrt{\mu}}^{\pi / \mu} e^{-\left(\mu^{2} / 2 \sigma^{2}\right) s^{2} d s=} & \lim _{t \rightarrow \infty} \sum_{|(t-n \mu) / \sqrt{t}|<\lambda} \frac{1}{\sqrt{2 \pi}} \\
& \cdot \frac{1}{\sigma \sqrt{n}} \exp \left\{\frac{-(t-n \mu)^{2}}{2 \sigma^{2} n}\right\} .
\end{aligned}
$$

This gives

$$
\lim _{t \rightarrow \infty} \sum_{n=1}^{\infty} \frac{1}{\sqrt{2 \pi}} \cdot \frac{1}{\sigma \sqrt{n}} \exp \left\{\frac{-(t-n \mu)^{2}}{2 \sigma^{2} n}\right\}=\frac{1}{\mu}
$$


Proof OF TheOReM 2.

$$
\begin{aligned}
& \lim _{t \rightarrow \infty} \sum_{n=1}^{\infty} \mid W_{t}(n)-\frac{\mu_{n}}{\sqrt{2 \pi}} \cdot \frac{1}{B_{n}} \exp \left\{\frac{-(t-n \mu)^{2}}{2 B_{n}^{2}}\right\} \mid \\
&< \lim _{t \rightarrow \infty} \sum_{n=1}^{\infty}\left|W_{t}(n)-\frac{\mu_{n}}{\sqrt{2 \pi}} \cdot \frac{1}{B_{n}} \exp \left\{\frac{-\left(t-A_{n}\right)^{2}}{2 B_{n}^{2}}\right\}\right| \\
&+\lim _{t \rightarrow \infty} \sum_{n=1}^{\infty} \mid \frac{\mu_{n}}{\sqrt{2 \pi}} \cdot \frac{1}{B_{n}} \exp \left\{\frac{-\left(t-A_{n}\right)^{2}}{2 B_{n}^{2}}\right\} \\
&-\frac{\mu_{n}}{\sqrt{2 \pi}} \cdot \frac{1}{B_{n}} \exp \left\{\frac{-(t-n \mu)}{2 B_{n}^{2}}\right\} \mid
\end{aligned}
$$

The first expression goes to 0 as $n \rightarrow \infty$ by Proposition 1. The second goes to 0 as $n \rightarrow \infty$ using Lemma 7 and Lemmas 1 and 8.

Proof of TheOREM 3.

$$
\lim _{\substack{t \rightarrow \infty \\ t \in R_{+}}}\left|P\left\{V_{t} \in A\right\}-\sum_{m=1}^{\infty} \frac{\alpha_{m}}{\mu_{m}} W_{t}(m)\right|=0
$$

by Theorem 1. By Theorem 2,

$$
\lim _{\substack{t \rightarrow \infty \\ t \in R_{+}}}\left|\sum_{m=1}^{\infty} \frac{\alpha_{m}}{\mu_{m}} W_{t}(m)-\sum_{m=1}^{\infty} \frac{\alpha_{m}}{\sqrt{2 \pi}} \cdot \frac{1}{B_{m}} \exp \left\{\frac{-(t-m \mu)^{2}}{2 B_{m}^{2}}\right\}\right|=0 .
$$

Let $\bar{\mu}_{m}=(\mu /(\alpha+1))\left(\alpha_{m}+1\right)$; hence $\mu /(\bar{\mu}+1)<\bar{\mu}_{m}<\mu(\bar{\mu}+1)$. Let $\bar{A}_{n}$ $=\sum_{k=1}^{n} \bar{\mu}_{k}$ and $\gamma_{n}=\sum_{k=1}^{n} \alpha_{k}$; therefore

$$
\frac{\bar{A}_{n}-n \mu}{\sqrt{n}}=\frac{1}{\sqrt{n}}\left[\frac{\mu}{(\alpha+1)} \gamma_{n}+\frac{n \mu}{\alpha+1}-n \mu\right]=\frac{\mu}{\alpha+1} \cdot \frac{\left(\gamma_{n}-\alpha n\right)}{\sqrt{n}} \rightarrow 0
$$

as $n \rightarrow \infty$. Next since $\bar{\sigma}^{2} n \geqslant B_{n}^{2} \geqslant(\underline{\sigma})^{2} n$ for all $n$ there is a subsequence $n_{k}$ such that $\sigma_{n_{k}}^{2} \geqslant(\underline{\sigma})^{2}$ for all $k$. We may construct an i.i.d. sequence $\left\{L_{n_{k}}\right\}_{k=1}^{\infty}$ of uniformly distributed random variables with mean 0 and variance $v$ where $\sqrt{3 v}<\frac{1}{2} \mu /(\bar{\mu}+1)$ (i.e., uniform on $[-\sqrt{3 v}, \sqrt{3 v}]$. Set $L_{n}=0$ off the subsequence. We may construct a sequence $\left\{Y_{n}\right\}_{n=1}^{\infty}$ of independent random variables, also independent of $\left\{L_{n_{k}}\right\}_{k=1}^{\infty}$, such that $Y_{n}$ has mean $\bar{\mu}_{n}$, has support on $\left(+\frac{1}{2} \mu /(\bar{\mu}+1), \infty\right)$ and such that the variance of $\bar{T}_{n}=Y_{n}+L_{n}$ is $\sigma_{n}^{2}$. It is quite easy to check that $\left\{\bar{T}_{n}\right\}_{n=1}^{\infty}$ satisfies Condition $\mathrm{C}(\mathrm{a})$ since $\bar{T}_{n_{k}}$ has a bounded density. By construction $\bar{T}_{n}$ is positive.

Applying Theorem 2 to $\left\{\bar{T}_{k}\right\}_{k=1}^{\infty}$ we have

$$
\lim _{t \rightarrow \infty} \sum_{m=1}^{\infty} \frac{\bar{\mu}_{m}}{\sqrt{2 \pi}} \cdot \frac{1}{B_{m}} \exp \left\{\frac{-(t-m \mu)^{2}}{2 B_{m}^{2}}\right\}=1 \text {. }
$$


Hence

$$
\lim _{t \rightarrow \infty} \sum_{m=1}^{\infty} \frac{\alpha_{m}+1}{\sqrt{2 \pi}} \cdot \frac{1}{B_{m}} \exp \left\{\frac{-(t-m \mu)^{2}}{2 B_{m}^{2}}\right\}=\frac{\alpha+1}{\mu} .
$$

But setting $0=\alpha=\alpha_{1}=\alpha_{2}=\ldots$ gives

$$
\lim _{t \rightarrow \infty} \sum_{m=1}^{\infty} \frac{1}{\sqrt{2 \pi}} \cdot \frac{1}{B_{m}} \exp \left\{\frac{-(t-m \mu)^{2}}{2 B_{m}^{2}}\right\}=\frac{1}{\mu},
$$

so that

$$
\lim _{t \rightarrow \infty} \sum_{m=1}^{\infty} \frac{\alpha_{m}}{\sqrt{2 \pi}} \cdot \frac{1}{B_{m}} \exp \left\{\frac{-(t-m \mu)^{2}}{2 B_{m}^{2}}\right\}=\frac{\alpha}{\mu} .
$$

This gives the result.

COROLlaRY 1. In the lattice case if Conditions $\mathrm{S}(\mathrm{a})-\mathrm{S}(\mathrm{c})$ are verified, then

$$
\lim _{\substack{t \rightarrow \infty \\ t \in R_{+}}} P\{\text { renewal at } t\}=\frac{1}{\mu} .
$$

Proof. Define $N_{t}=\inf \left\{n-1 \mid S_{n-1} \leqslant t<S_{n}\right\}$.

The process $V_{t}=t-S_{N_{t}}, t \in R_{+}$, is a nonstationary regenerative process which regenerates itself when $V_{t}=0$. Also $V_{t}=0$ if and only if $S_{N_{t}}=t$. The mean time $V_{t}=0$ per cycle is 1 , so applying Theorem 3

$$
\lim _{\substack{t \rightarrow \infty \\ t \in R_{+}}} P\{\text { renewal at } t\}=\frac{1}{\mu}
$$

ACKNOWLEDGEMENTs. I thank Cornell University for its hospitality and Professor Kesten for his help.

\section{BIBLIOGRAPHY}

1. D. McDonald, On local limit theorems for integer valued random variables, Teor. Verojastnost. i Primenen. (to appear).

2. On semi-Markov and semi-regenerative processes. I, Z. Wahrscheinlichkeitstheorie und verw. Gebiete 42 (1978), 261-277.

3. __ On semi-Markov and semi-regenerative processes. II, Ann. Probability 6 (1978), 995-1014.

4. J. Mineka, A criterion for tail events for sums of independent random variables, $\mathbf{Z}$. Wahrscheinlichkeitstheorie und verw. Gebiete 25 (1973), 163-170.

5. L_ Local limit theorems and recurrence conditions for sums of independent integer-valued random variables, Ann. Math. Statist. 43 (1972), 251-259.

6. A. B. Muhin, On local limit theorems for densities and asymptotic uniform distributedness, Izv. Akad. Nauk UzSSR, Ser. Fiz.-Mat. Nauk 15 (1971), 17-23. (Russian)

7. S. Orey, Tail events for sums of independent random variables, J. Math. Mech. 15 (1966), 937-951.

8. W. L. Smith, Renewal theory and its ramifications, J. Roy. Statist. Soc. Ser. B 20 (1958).

Department of Mathematics, University of Ottawa, Ontario, Canada 